\title{
New Algorithms for Processing Signals at the Receiver Side for Wireless Communication Systems with Massive MIMO Technology
}

\author{
Aleksey E. Smirnov \\ Department of Information Security \\ Moscow Technical University of Communications and Informatics \\ Moscow, Russia \\ smirnov.al.ed@gmail.com
}

\begin{abstract}
Multiple-Input Multiple-Output systems (MIMO) have been a trending direction of research in the past few years. MIMO technology offers increased throughput compared to single-antenna systems. Extension of MIMO systems is a largescale MIMO (or massive MIMO) that use antenna arrays with a few hundred antennas. Therefore, the number of operations required for computation of the estimate of the vector of transmitted symbols is increasing with the increased number of antenna arrays. The Minimum Mean Square Error (MMSE) detection technique is the basic one for MIMO systems due to low computational expenses as compared with the Maximum Likelihood (ML) method and a higher resistance to noise as compared to Zero Forcing (ZF) technique. Unfortunately, MMSE demodulation technique cannot be realized for massive MIMO systems due to high level of computational expenses.
\end{abstract}

The paper provides us description of new low-complexity as compared to MMSE iterative demodulation technique for massive MIMO systems. To compare a resistance to noise of new iteration demodulation technique with MMSE technique it is developed a simulation model.

Key words: MIMO, detection, computational complexity, Neumann series, iterative detector.

\section{INTRODUCTION}

The problem of the received signal demodulation in the multi-array systems at the receiver end at the known matrix of the channel is one of the traditional aspects in the sphere of wireless telecommunication. The problem consists in recovering of the transmitted signal at the receiver end from the received vector of counts at the known channel matrix and at known statistical characteristics of the noise [1].

There is a certain number of known algorithms used for the purpose of demodulation, whereas each of the algorithms has its own advantages and drawbacks as compared to the other. These algorithms allow computing the estimate of the vector of transmitted symbols at the receiver end. Using some of them allows obtaining high accuracy of the estimate, however, at that, as a consequence, there occurs high complexity of the signal processing. On the contrary, others possess a lower complexity but the estimate turns out to be less accurate. Depending upon specific conditions and criteria at developing of the telecommunication system, it is necessary to make a choice in favor of one of the algorithms.

\section{SYSTEM MODEL}

An abstract model of the MIMO system based on which different configurations of the system can be built (Fig. 1).

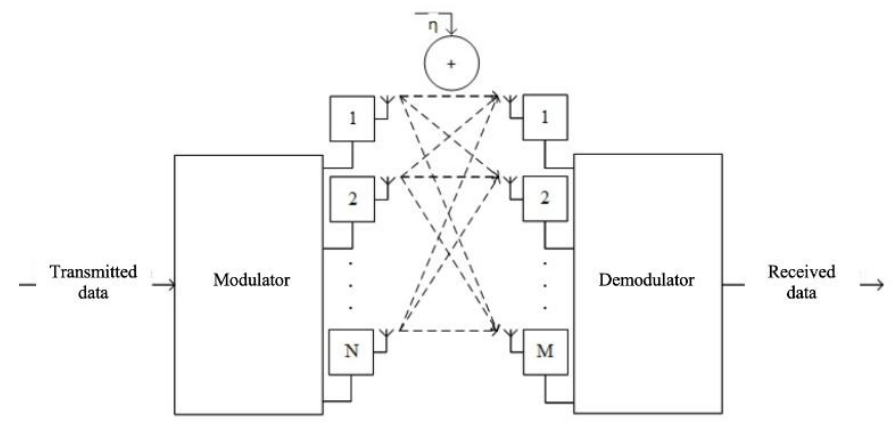

Figure 5. The MIMO system model

Here $N$ is the number of antennas at the transmitter part, $M$ is the number of antennas at the receiver end. At the receiver end, the signals from each of the transmitting antennas are emitted simultaneously and within one and the same frequency band. In the channel these signals are subject to the influence of the Rayleigh fading and the additive Gaussian white noise. Therefore, an additive aggregate of $N$ transmitted signals arrives at each of $M$ receiving arrays.

Mathematical model of the MIMO system has the following representation:

$$
\mathbf{y}=\mathbf{H} \cdot \mathbf{x}+\boldsymbol{\eta},
$$

where $y$ is the vector of received complex counts with the dimensionality $M \times 1 ; \quad \mathbf{H}$ is the matrix of the telecommunication channel complex transmission coefficients with the dimensionality $M \times N, \mathbf{x}$ is the vector of transmitted informational symbols with the dimensionality $N \times 1 ; \boldsymbol{\eta}$ is the complex random Gaussian noise vector in the telecommunication channel with the dimensionality $M \times 1$.

Three main techniques used for computing the estimate of transmitted symbols [1] are the following:

- ZF;

- MMSE;

- ML.

\section{A. Zero Focrcing $(Z F)$}

$\mathrm{ZF}$ demodulator is the best linear demodulation technique in terms of complexity but suffers from noise enhancement. Using this demodulator, the estimate is computed under the equation: 


$$
\hat{\mathbf{x}}_{Z F}=\mathbf{G}_{Z F} \cdot \mathbf{y}=\left(\mathbf{H}^{\prime} \mathbf{H}\right)^{-1} \cdot \mathbf{H}^{\prime} \cdot \mathbf{y}
$$

In order to compute estimate of transmitted symbols using $\mathrm{ZF}$, it is considered the matrix of the channel only but the availability of the noise is disregarded that results in decreasing of the resistance to noise.

\section{B. Minimum Mean Square Error (MMSE)}

MMSE is introduced to overcome the negative effect of noise enhancement. This algorithm provides better estimate computation results because availability of the noise is taken into consideration. The estimate obtained as the result of the MMSE demodulator operating execution is represented mathematically as follows:

$$
\hat{\mathbf{x}}_{M M S E}=\mathbf{G}_{M M S E} \cdot \mathbf{y}=\left(\mathbf{H}^{\prime} \mathbf{H}+2 \sigma^{2} \mathbf{1}\right)^{-1} \cdot \mathbf{H}^{\prime} \cdot \mathbf{y}
$$

where $2 \sigma^{2}$ is the aggregate dispersion of the real and imaginary components of the Gaussian noise vector and $I$ is identity matrix. C. Maximum Likelihood (ML)

The ML method possesses the best characteristics of resistance to noise [1]. This demodulation technique minimizes the difference between $y$ and $x$ distorted by the channel. The estimate obtained with the help of this method minimizes the square of the discrepancy norm:

$$
\hat{\mathbf{x}}_{M L}=\arg \min _{\mathbf{x} \in \mathbf{X}^{N}}\|\mathbf{y}-\mathbf{H} \cdot \mathbf{x}\|^{2}
$$

\section{COMPUTATIONAL COMPLEXITY}

The mathematical algorithms can be classified [1] in accordance with complexity of their execution. Considering that the algorithm is composed of a sequence of strictly determined operations (commands) [1], the algorithms can be classified depending upon the number of operations required for obtaining of the result. The parameter, which is called the computational complexity, is often used for classification of the algorithms. The term is used for determining of the number of elementary arithmetical operations, which must be performed for obtaining the solution to a certain problem.

Let us consider in detail the detection algorithms mentioned above. We shall regard the telecommunication systems, in which the number of arrays at the receiver end and the number of arrays at the transmitter end are equal; that is $N=M$. The number of elementary arithmetical operations required for obtaining of the estimate $\hat{\mathbf{x}}_{M M S E}$ and $\hat{\mathbf{x}}_{Z F}$ depends primarily upon the number of arrays at the received end and at the transmitted part that is upon the dimension of the channel matrix. To find the minimum, according to (4), it is necessary to perform enumeration upon all the possible combinations of the vector of complex informational symbols $\mathbf{x}$. The number of operations depends upon two parameters of the system such as the number of transmitting arrays and the type of modulation used.

Considering the trend to growth of the data transmission rate in the present-day telecommunication systems, signal processing at the receiver end has to be performed [1] during the interval of time equal to the duration of the informational symbol. With the increase of the number of the receiving and transmitting arrays and modulation type the number of mathematical operations, which are required for obtaining of the estimate of the vector of transmitted symbols, is also increasing. Due to the fact, that implementation of the ML method requires execution of a great number of operations, obtaining of the estimate for the vector of transmitted symbols using the ML method cannot be realized practically [1].

Development of wireless communication systems resulted [3] in origination of large-size MIMO systems, or the so-called massive MIMO. Their main difference from the existing multiarray systems is in availability of the antenna arrays, the number of arrays in which exceeds by several times the number of arrays in traditional systems [3][3]. In this condition, an apparent drawback as compared with the traditional multi-array systems is in the fact that increasing of the number of arrays at the receiver and transmitter ends results in increasing of the channel matrix dimensionality, and, consequently, in increasing of the number of mathematical operations [5] necessary for obtaining of the estimate at demodulation. Therefore, with the increased number of arrays the computational complexity while obtaining the estimates $\hat{\mathbf{x}}_{Z F}$ and $\hat{\mathbf{x}}_{M M S E}$ is increasing, however, the advantage of a low complexity as compared with obtaining of the estimate $\hat{\mathbf{x}}_{M L}$ is preserved.

\section{COMPLEXITY OF MMSE DETECTOR}

We address to the equation (3) in order to obtain the estimate of MMSE. We shall separately consider all the operations used for obtaining the estimate upon the MMSE algorithm:

- multiplication of the matrix with the dimensionality $N \times N$ by the matrix with the dimensionality $N \times N$;

- multiplication of the matrix with the dimensionality $N \times N$ by the vector-column with the dimensionality $N$ (this operation has to be performed twice);

- summation of two matrices with the dimensionality $N \times N$;

- inversion of the matrix with the dimensionality $N \times N$ with the help of solving of the system of linear algebraic equations (SLAE).

Main contribution in overall computational complexity for obtaining of the estimate $\hat{\mathbf{x}}_{M M S E}$ consists in the operation of multiplication of two matrices and the operation inversion of the matrix.

It is important to notice, that the channel matrix and the vector of received counts contain complex numbers. Therefore, total number of elementary arithmetical operations required for obtaining of the estimate for real multiplications and two real summations is increasing.

\section{ITERATIVE DETECTOR FOR MASSIVE MIMO}

To recover of the transmitted signal at the receiver end from the received vector in wireless communication systems based on massive MIMO technology, we propose a new iteration detector with lower computational complexity as compared with known MMSE detector. 
Let $\mathbf{T}=\left(\mathbf{H}^{\prime} \mathbf{H}+2 \sigma^{2} \mathbf{1}\right)$ and $\mathbf{Y}=\mathbf{H}^{\prime} \cdot \mathbf{y}$, next step we multiple left and right side (3) by $\mathrm{T}$ and the estimate of the vector of transmitted is represented as follows:

$$
\mathbf{T} \cdot \hat{\mathbf{x}}_{\text {MMSE }}=\mathbf{Y}
$$

Then the process of obtaining of the estimation $\hat{\mathbf{x}}_{\text {MMSE }}$ at the receiver end consists in solving of the given system of linear equations (SOLE). There are so many different methods of solving the SOLE [6]Ошибка! Источник ссылки не найден.. One part of them are direct methods: provide an exact solution. These methods have high computational complexity. The second ones are indirect solver. Direct methods for obtaining exact solution of SOLE have the same computational complexity as compared to obtaining of the estimation $\hat{\mathbf{x}}_{\text {MMSE }}$ according to equation (3).

To decrease computational complexity for signal processing at the receiver side, we propose to use iterative methods for solving SOLE (5). Using these methods, it is necessary to start with a first approximation and compute iteratively sequence of approximation without ever reaching exact solution. In this case, we have an opportunity to reduce number of iterations for solving SOLE and, consequently, decrease of the number of mathematical operations necessary for obtaining of the estimate at demodulation. The main goal while choosing the best iterative methods for solving particular SOLE is in finding compromise between complexity and accuracy of iterative method.

The stop criteria for these methods are the maximum number of iterations or defined small value of error $\varepsilon<\max _{1 \leq i \leq N}\left\|\hat{\mathbf{x}}_{P R O P}^{i+1}-\hat{\mathbf{x}}_{P R O P}^{i}\right\|$ and we can rewrite equation (5) as:

$$
\mathbf{T} \cdot \hat{\mathbf{x}}_{\text {PROP }} \approx \mathbf{Y}
$$

where $i$ is the iteration number; $j=1,2 \ldots N$ is the index of vector $\hat{\mathbf{x}}_{P R O P}$ component.

To decrease computational complexity of obtaining of the estimation $\hat{\mathbf{x}}_{P R O P}$ at the receiver end a lot, it is possible to use iterative methods to solve the SOLE (6) with maximum number of iterations $L=\frac{N}{4}$.

We propose to use iterative BiConjugate Gradient Stabilized (l) (BiCGstab(1)). This approach is described in [7] [8]. To achieve a maximum effect in decreasing computational complexity for obtaining estimation $\hat{\mathbf{x}}_{P R O P}$ the maximum number of iterations $L$ should be smaller than matrix $\mathbf{H}$ dimensions $N$ for few times $L<<N$.

\section{SimULATION RESULTS}

To compare the results of noise immunity of different demodulation techniques for massive MIMO systems, we perform a simulation model. Simulation parameters are in Table.

\section{Simulation parameters}

\begin{tabular}{|l|c|}
\hline Parameter & Value \\
\hline Channel & MIMO rayleigh fading channel \\
\hline
\end{tabular}

\begin{tabular}{|l|l|}
\hline Parameter & \multicolumn{1}{|c|}{ Value } \\
\hline $\begin{array}{l}\text { Number of } \\
\text { antennas on } \\
\text { BS side }\end{array}$ & 64 \\
\hline $\begin{array}{l}\text { Number of } \\
\text { antennas on } \\
\text { user side }\end{array}$ & 64 \\
\hline $\begin{array}{l}\text { Transceiver } \\
\text { architecture }\end{array}$ & V-BLAST \\
\hline $\begin{array}{l}\text { Error } \\
\text { correcting } \\
\text { code type }\end{array}$ & Convolutional coding (rate 1/2) \\
\hline $\begin{array}{l}\text { Modulation } \\
\text { type }\end{array}$ & 16 -QAM \\
\hline $\begin{array}{l}\text { Total number } \\
\text { of iterations } \\
\text { for solving } \\
\text { SOLE }\end{array}$ & 16 \\
\hline
\end{tabular}

And as we use convolutional coding in our system, we have one more operation at the receiver side that requests cubic level of computational complexity matrix inversion for calculating correlation matrix for MMSE despite we need to know only main diagonal for soft decision demodulation based on loglikelihood ratio. Its operation describes the following equation:

$$
\mathbf{R}_{M M S E}=\left(\mathbf{H}^{\prime} \mathbf{H}+2 \sigma^{2} \mathbf{I}\right)^{-1} \cdot 2 \sigma^{2}
$$

Obtaining correlation matrix for MMSE requires more elementary arithmetical operations than obtaining solution of SOLE (6) using BiCGstab(1). We have no need to calculate each element of matrix $\mathbf{R}_{\text {MMSE }}$. To avoid direct matrix inversion, we propose to use approximation based on Neumann series. This way we can reduce computational expenses for this operation. Use of Neumann series for decreasing computational complexity of matrix inversion is described in [9].

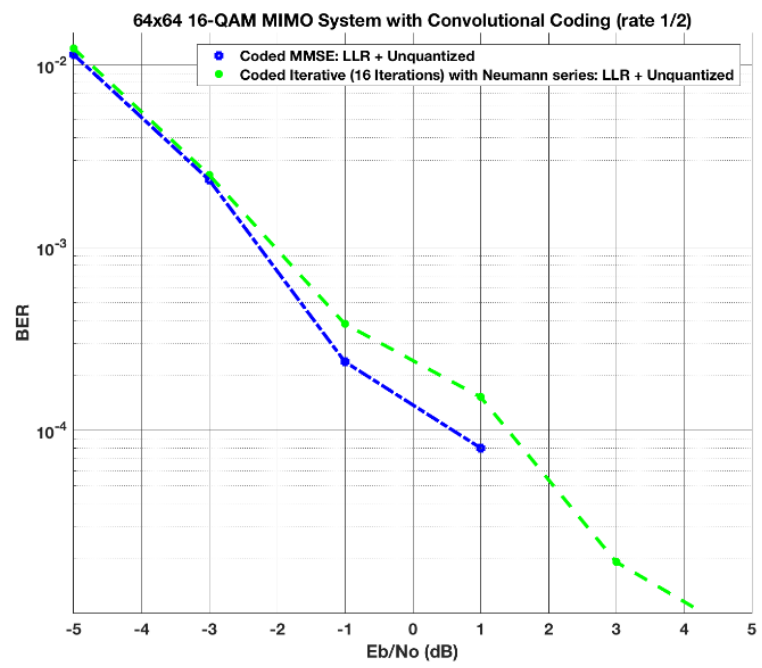

Fig. 6. Noise immunity comparison for MMSE and iterative detectors

The results of simulation (Fig. 2) show that application of iterative detector combined with Neumann series approach for signal processing at the receiver end allows decreasing a total number of the required elementary operations for 5,5 times with 
losing in the resistance to noise (about $0,2 \mathrm{~dB}$ for $\mathrm{BER}=10^{-3}$ ) for the matrices of complex transmission coefficients of the telecommunication channel with the large dimensionality, which can be found in the promise massive MIMO systems.

\section{REFERENCES}

[1] Бакулин М.Г., Варукина Л.А., Крейнделин В.Б. Технология MIMO: принципы и алгоритмы. М.: Горячая линия Телеком, 2014. [Bakulin M.G., Varukina L.A., Kreyndelin V.B. MIMO technology: principles and algorithms. Moscow: Hot line Telecom, 2014].

[2] Levitin A.V. Introduction to the design and analysis of algorithms. Reading: Addison-Wesley, 2007.

[3] Larsson E.G., Edfors O., Tufvesson F.et al. Massive MIMO for next generation wireless systems // Communications Magazine, IEEE. 2014. Vol. 52, N 2. P. 186-195.

[4] Rusek F., Persson D., Buon Kiong Lau, et al. Scaling up MIMO: challenges with very large arrays // Signal Proccesing Magazine, IEEE. 2013. Vol. 30, N 1. P. 40-60.

[5] Крейнделин В.Б., Смирнов А.Э. Снижение вычислительной сложности алгоритмов демодуляции в многоантенных системах за счёт использования быстрых алгоритмов // Telecommunications and Radio Engineering. 2016. Том. 75, N 19. C. 1757-1773. [Kreyndelin V.B., Smirnov A.E. Decreasing of computational complexity of demodulation algorithms in multiantenna systems due to application of fast algorithms // Telecommunications and Radio Engineering. 2016. Vol. 75, N 19. P. 1757-1773].

[6] Самарский А.А., Гулин А.В, Численные методы. М.: Наука, 1989. [Samarskii A.A., Gulin A.V. Numerical methods. Moscow: Nauka, 1989].

[7] Sleijpen G.L.C., van der Vorst H.A., Fokkema D.R. BiCGstab(1) and other hybrid Bi-CG methods // Numerical Algorithms. 1994. Vol. 7. P. 75-109.

[8] Sleijpen G.L.C., Fokkema D.R. BiCGstab(1) for linear equations involving unsymmetric matrices with complex spectrum // Electronic Transactions on Numerical Analysis. 1993. Vol. 7. P. 1132.

[9] Monteiro F. A., Rosário F., Rodrigues A. Fast matrix inversion updates for massive MIMO detection and precoding // Signal Proccesing Letters, IEEE. 2016. Vol. 23, N 1. P. 75-79. 